\title{
A Displacement Based Analytical Model to Determine Residual Stress Components in a Finite Elastic Thin Plate with Hole-Drilling Method
}

\author{
Yuming Li', Boussad Abbes ${ }^{1}$, Fazilay Abbes ${ }^{1}$, \\ Yingqiao Guo ${ }^{1}$, Xiaolu Gong ${ }^{2}$ \\ ${ }^{1}$ Lab. GRESPI/Mécanique, Université de Reims Champagne-Ardenne, Reims, France \\ ${ }^{2}$ Université de Technologie de Troyes, Troyes, France \\ Email: yuming.li@univ-reims.fr
}

How to cite this paper: Li, Y.M., Abbes, B., Abbes, F., Guo, Y.Q. and Gong, X.L. (2018) A Displacement Based Analytical Model to Determine Residual Stress Components in a Finite Elastic Thin Plate with Hole-Drilling Method. Journal of Applied Mathematics and Physics, 6, 1571-1580. https://doi.org/10.4236/jamp.2018.68133

Received: June 4, 2018

Accepted: August 6, 2018

Published: August 9, 2018

\begin{abstract}
In order to measure the residual stress components in an elastic thin plate, the hole-drilling strain-gage method has been used. This method enables to determine the relation between the magnitudes and directions of the principal stresses and the strain relaxation about the hole. In the existing analytical models based on stress field, the formulations associated with the hole-drilling method are based on the assumption of an infinite plate, this may cause some errors for a finite plate and it's difficult to validate these solutions by FE methods. Furthermore, in the composite, the displacement field is continuous but the stress field is not necessarily continuous, the displacement field based method has to be used. In the present paper an analytical model based on a displacement field described by a function with coefficients to determine for a finite round thin plate is presented. The coefficients used in the displacement field are independent on the three residual stress components, and they are determined by minimization of the internal strain energy during the hole-drilling process. Once the coefficients in the displacement field are determined, three strains measured in three radial directions are utilized to determine the three residual stress components. The proposed analytical model can be also adapted to infinite plate by assuming that the diameter of the round plate tends to infinite.
\end{abstract}

\section{Keywords}

Residual Stress, Hold Drilling, Displacement Based, Internal Strain Energy, Analytical Model 


\section{Introduction}

Residual stresses represent stresses that remain in a solid material when the original cause of the stresses has been removed. They are generated in the solid material after manufacturing processes such as casting, welding, machining, heat treatment, etc.

In order to measure the residual stresses in a thin plate, the hole-drilling method has been used experimentally [1] by using the strain-gages and theoretically [2] [3]: during the hole-drilling in the plate, the strains occurred in three different directions enables to determine the residual stress components in the plate. Some analytical solutions [4] [5] [6] based on stresses for an infinite plate have been proposed. Meanwhile it's difficult to use FE method to validate these solutions for two reasons: FE method is displacement based and for finite dimensions.

In the present paper, a displacement field based analytical model for a finite round thin plate is proposed. The proposed displacement field is expressed by three residual stress components and some coefficients to determine. These coefficients are independent of the three residual stress components. The minimization of the internal strain energy enables to determine these coefficients. Once these coefficients are determined, three strains in three radial directions are used to determine the residual stress components.

The minimization of internal strain energy replaces the force equilibrium resolution and a flexible displacement field function can adapt very well to the boundary conditions. This displacement based model has been validated by FE method in ABAQUS and it is also adapted for infinite plate mathematically.

\section{Problem Description}

Hole-drilling method is to determine the residual stress in a thin plate by measuring the strains in three different radial directions occurred during the hole-drilling presented in Figure 1. The stress status is supposed to be plane-stress

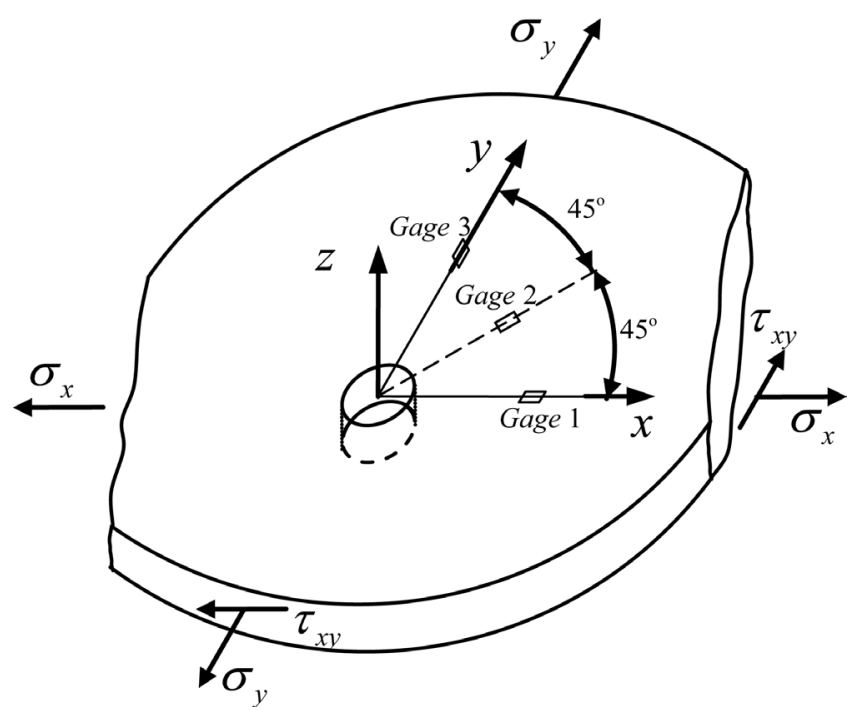

Figure 1. Residual stress measurement by hole-drilling method. 
due to two facts: the plate is thin and the dimension of the plate is much larger than the drilled hole. All the stresses in the out-plane direction are negligible.

The plate is supposed large enough, thus a round plate is taken as an example to be treated even for other different forms of the plate. Indeed, all the specimen for the strain measurement can be fixed by a large circle perimeter and the outer bound becomes a round surface.

The residual stress components in the plate are given by:

$$
\left\langle\sigma_{0}\right\rangle=\left\langle\sigma_{x 0} \sigma_{y 0} \sigma_{x y 0}\right\rangle
$$

In order to determine the above three residual stress components, the relation between the three measured strains in three radial directions and the three residual stress components will be investigated. In the literature [1], this has been accomplished by means of an empirically determined relation between the magnitudes and directions of the principal stresses and the strain relaxation about the hole during the hole-drilling process.

\section{Displacement Field Based Model with Minimization of Internal Strain Energy}

In the experimental measurement, the strains are measured in three radial directions, thus the residual stress vector is expressed in the cylindrical coordinates as follows by using coordinates transformation:

$$
\left\{\sigma_{0}\right\}_{P}=\left\{\begin{array}{l}
\sigma_{r 0} \\
\sigma_{\theta 0} \\
\sigma_{r \theta 0}
\end{array}\right\}=\left\{\begin{array}{l}
\sigma_{c}+\sigma_{n} \\
\sigma_{c}-\sigma_{n} \\
\sigma_{\tau}
\end{array}\right\}
$$

where $\sigma_{c}=\frac{\sigma_{x 0}+\sigma_{y 0}}{2}, \sigma_{n}=\sigma_{\Delta} \cos 2 \theta+\sigma_{x y 0} \sin 2 \theta$,

$$
\sigma_{\tau}=-\sigma_{\Delta} \sin 2 \theta+\sigma_{x y 0} \cos 2 \theta, \sigma_{\Delta}=\frac{\sigma_{x 0}-\sigma_{y 0}}{2} .
$$

\subsection{Displacement, Strain and Stress Fields in a Thin Plastic Plate}

By the analysis and observation of the results obtained by using FE method in $\mathrm{ABAQUS}^{\circledR}$, the displacement field is proposed by a polynomial of $\xi^{-(2 i-1)}$ with coefficients to determine $A_{p} B_{i}$ and $C_{i}$ and with three residual stress components, as well as the angle $\theta$.

$$
\left\{\begin{array}{l}
u_{r}=\sum_{i=0}^{N_{C}} C_{i} \frac{a}{E} \sigma_{c} \xi^{-j}+\sum_{i=0}^{N_{A}} A_{i} \frac{a}{E} \sigma_{n} \xi^{-j} \quad \text { with } \xi=\frac{r}{a}, j=2 i-1 \\
u_{\theta}=\sum_{i=0}^{N_{B}} B_{i} \frac{a}{E} \sigma_{\tau} \xi^{-j}
\end{array}\right.
$$

where $a$ is the drilled hole's radius, $E$ is the Yong's Module; $N_{A}, N_{B}$ and $N_{C}$ define the degrees of the polynomial in the displacement; $C_{D} A_{i}$ and $B_{i}$ are coefficients to determine and they are supposed to be independent of the residual stress components, Additionally $\sigma_{c}$ and $\sigma_{n}$ in $u_{r}$ are independent one another as $u_{r}$ holds for any choice of the residual stresses. 
The three cylindrical components of the strain field $\{\varepsilon\}_{P}$ are given by the follows:

$$
\varepsilon_{r}=\frac{\partial u_{r}}{\partial r} ; \varepsilon_{\theta}=\frac{1}{r} \frac{\partial u_{\theta}}{\partial \theta}+\frac{u_{r}}{r} ; \gamma_{r \theta}=\frac{1}{r} \frac{\partial u_{r}}{\partial \theta}+\frac{\partial u_{\theta}}{\partial r}-\frac{u_{\theta}}{r}
$$

By using Equations (3) and Equation (4a), the strain in the radial direction $\varepsilon_{r}$ can be expressed as follows:

$$
\varepsilon_{r}=-\sum_{i=0}^{N_{C}} j C_{i} \frac{\sigma_{c}}{E} \xi^{-2 i}-\sum_{i=0}^{N_{A}} j A_{i} \frac{\sigma_{n}}{E} \xi^{-2 i}, \text { with } j=2 i-1
$$

Once the coefficients used in the displacements field are determined, Equation (4b) can be used to determine the three residual stress components by using three measured strain in three radial directions.

The hole-drilling process is supposed to be a plane stress problem, thus the final stress state after drilling can be calculated by the following:

$$
\left\{\sigma_{1}\right\}_{P}=\left\{\sigma_{0}\right\}_{P}+[C]\{\varepsilon\}_{P}
$$

with $[C]=\frac{E}{1-v^{2}}\left[\begin{array}{ccc}1 & v & 0 \\ v & 1 & 0 \\ 0 & 0 & (1-v) / 2\end{array}\right]$

By using Equation (4) and Equation (5), the components of the final stress can be expressed as follows:

$$
\left\{\begin{array}{l}
\sigma_{r 1}=\sigma_{c}+\frac{1}{1-v^{2}} \sum_{i=0}^{N_{C}}(-j+v) C_{i} \xi^{-2 i} \sigma_{c}+\sigma_{n}+\frac{1}{1-v^{2}}\left(\sum_{i=0}^{N_{A}}(-j+v) A_{i} \xi^{-2 i}-2 v \sum_{i=0}^{N_{B}} B_{i} \xi^{-2 i}\right) \sigma_{n} \\
\sigma_{\theta 1}=\sigma_{c}+\frac{1}{1-v^{2}} \sum_{i=0}^{N_{C}}(-v j+1) C_{i} \xi^{-2 i} \sigma_{c}-\sigma_{n}+\frac{1}{1-v^{2}}\left(\sum_{i=0}^{N_{A}}(-v j+1) A_{i} \xi^{-2 i}-2 \sum_{i=0}^{N_{B}} B_{i} \xi^{-2 i}\right) \sigma_{n} \\
\sigma_{r \theta 1}=\sigma_{\tau}+\frac{1}{(1+v)} \sum_{i=0}^{N_{A}} A_{i} \xi^{-2 i} \sigma_{\tau}-\frac{1}{2(1+v)} \sum_{i=0}^{N_{B}}(1+j) B_{i} \xi^{-2 i} \sigma_{\tau} \\
\quad \text { with } j=2 i-1
\end{array}\right.
$$

\subsection{Displacement and Stress Boundary Conditions}

In the thin elastic plate, two boundary conditions are used:

1) For the assumption that the outer bound is very large with respect to the drilled hole, at the outer bound of the circle plate $(\xi=\lambda)$, the displacements $u_{r}$ and $u_{\theta}$ are null. Furthermore, $\sigma_{c}$ and $\sigma_{\sigma}$ are independent, with Equation (3) the following relation is held:

$$
\sum_{i=0}^{N_{C}} C_{i} \lambda^{-2 i+1}=0 ; \sum_{i=0}^{N_{A}} A_{i} \lambda^{-2 i+1}=0 ; \sum_{i=0}^{N_{B}} B_{i} \lambda^{-2 i+1}=0
$$

For a finite plate, $\lambda$ is given by the plate's dimension. For infinite plate, $\lambda \rightarrow \infty$ and Equation (7) gives:

$$
C_{0} \rightarrow 0 ; A_{0} \rightarrow 0 ; B_{0} \rightarrow 0
$$

It is worth mentioning that the values the above three coefficients tend to zero 
does not mean that they can be directed eliminated from the displacement field function, because their derivatives about other coefficients are not zero which are used in the internal strain energy.

2) In the inner free boundary at the hole, the radial stress and the shearing stress in the plane are null.

$$
\sigma_{r 1}=\sigma_{r \theta 1}=0 \quad(\xi=r / a=1)
$$

which gives the following with the independence between $\sigma_{c}$ and $\sigma_{\sigma}$ :

$$
\left\{\begin{array}{l}
\sum_{i=0}^{N_{C}}(-j+v) C_{i}=-\left(1-v^{2}\right) \\
\sum_{i=0}^{N_{A}}(-j+v) A_{i}-2 v \sum_{i=0}^{N_{B}} B_{i}=-\left(1-v^{2}\right) \\
\sum_{i=0}^{N_{A}} A_{i}-\frac{1}{2} \sum_{i=0}^{N_{B}}(1+j) B_{i}=-(1+v)
\end{array}\right.
$$

These two boundary conditions provide six equations which allows the six coefficients $C_{0}, C_{1}, A_{0}, A_{1}, B_{0}$ and $B_{1}$ to be dependent with other coefficients. And by using Equation (10) their derives by $C_{i}, A_{i}$ and $B_{i}$ can be calculated. These derives will be always kept in the calculations even for the infinite case $\lambda \rightarrow \infty, C_{0} \rightarrow 0 ; A_{0} \rightarrow 0 ; B_{0} \rightarrow 0$.

\subsection{Coefficient of Displacement Functions Settings}

1) Choice of $C_{i}$

The coefficients $C_{i}$ concerns $\sigma_{c}$ that is the average residual normal stress. Due to its smooth change of the average residual normal stress, $N_{C}=1$ is proposed. The parts concerning $C_{i}$ in Equation (7) and Equation (10) given in the two boundary conditions can be expressed as follows:

$$
\left\{\begin{array}{l}
C_{0} \lambda+C_{1} \lambda^{-1}=0 \\
(1+v) C_{0}+(-1+v) C_{1}=-\left(1-v^{2}\right)
\end{array}\right.
$$

The above equation gives the coefficients $C_{0}$ and $C_{1}$. And for the infinite plate, $\lambda \rightarrow \infty$ which gives the same expression as in the Kirsch's equation [5].

2) Choice of $A_{i}$ and $B_{i}$

$N_{A}=2, N_{B}=2$ are the minimum for the calculation. That doesn't avoid other choices of these two parameters. $A_{i}$ and $B_{i}$ are set as unknown variables ( $i>1)$. By using the parts concerning $A_{i}$ and $B_{i}$ in Equations ((7) and (10)) given by the two boundary conditions, the coefficient derives about $A_{i}$ and $B_{i}$, that is, $\frac{\partial A_{k}}{\partial A_{i}}, \frac{\partial B_{k}}{\partial A_{i}}, \frac{\partial A_{k}}{\partial B_{i}}, \frac{\partial B_{k}}{\partial B_{i}}(k=0,1)$ can be calculated:

$$
\left\{\begin{array}{l}
\sum_{i=0}^{N_{A}} A_{i} \lambda^{-2 i+1}=0 ; \sum_{i=0}^{N_{B}} B_{i} \lambda^{-2 i+1}=0 \\
\sum_{i=0}^{N_{A}}(-j+v) A_{i}-2 v \sum_{i=0}^{N_{B}} B_{i}=-\left(1-v^{2}\right) ; \sum_{i=0}^{N_{A}} A_{i}-\frac{1}{2} \sum_{i=0}^{N_{B}}(1+j) B_{i}=-(1+v)
\end{array}\right.
$$




\subsection{Coefficients Determination by Internal Strain Energy Minimization}

The coefficients $A_{i}$ and $B_{i}$ to determine in the displacement field can be obtained the minimizing the internal strain energy of the circle plate during the hole-drilling process. The internal strain energy for a determined circle plate can be given by follows:

$$
\begin{aligned}
& \phi_{\text {int }}=\frac{1}{2} \iiint_{V}\left(\left\langle\sigma_{1}\right\rangle_{P}+\left\langle\sigma_{0}\right\rangle_{P}\right)\left\{\varepsilon_{1}\right\} d V \\
& =\frac{t}{2} \int_{\theta=0}^{\theta=2 \pi} \int_{r=a}^{r=\lambda a}\left(\left\langle\sigma_{1}\right\rangle_{P}+\left\langle\sigma_{0}\right\rangle_{P}\right)[C]^{-1}\left(\left\{\sigma_{1}\right\}_{P}-\left\{\sigma_{0}\right\}_{P}\right) r d r d \theta
\end{aligned}
$$

where $t$ is the thickness of the plate and the minimization of the internal strain energy is translated by:

$$
\frac{\partial \phi_{\text {int }}}{\partial A_{i}}=0 ; \frac{\partial \phi_{\text {int }}}{\partial B_{i}}=0 \quad(i>1)
$$

Equation (12) and Equation (14) form a linear system of the coefficients $A_{i}$ and $B_{i^{*}}$ A linear resolution is used to solve the equations.

$\lambda \rightarrow \infty$ in Equation (13) and Equation (14) gives the solution for the infinite plate. As there are some infinite-value terms in the linear system of equations, the limits of the expression possessing infinite-value terms have been calculated and simplified in the deductions.

\section{Numerical Validation of Analytical Displacement Based Model}

The results obtained by FE simulations in ABAQUS using the brick elements are used to validate the analytical model. The investigation of the displacement field used in the analytical model and that in the FE simulations in ABAQUS is presented hereafter.

The numerical simulation in ABAQUS of the hole-drilling process has been fulfilled by two steps:

1) Step 1: initial settings

The residual stress field $\left\langle\sigma_{x 0}, \sigma_{y 0}, \tau_{x y 0}\right\rangle$ in all the plate is predefined and will remain unchanged during the simulation; the displacements in the vertical surface of the plate's outer bound and the drilled hole are set zero.

2) Step 2: hole drilling process settings

Disable the fixed displacements setting on the vertical surface of the drilled hole. In this phase, the plate will be deformed with the constraints of the outer bound. The equilibrium of the plate represents the end of the hole drilling process.

The above settings have shown the differences of displacement based model and stress based model. In the displacement based model, the displacements are used as unknown variables and it's easy to be fulfilled by FE method.

Two cases of residual stress have been taken under investigation: 1) $\sigma_{x 0}=20$ Mpa; 2) $\tau_{x y 0}=20 \mathrm{Mpa}$ in order to simulate the tensile and shearing effects of 
the residual stresses individually. All the other cases can be considered as combined cases of the tensile and shearing effects. The geometrical parameters of plate used in Figure 2 are given as follows: drilled hole radius $a=5 \mathrm{~mm}$, radius of the plate $\lambda a=80 \mathrm{~mm}$, thickness of the plate $t=5 \mathrm{~mm}$. The Yong's module $E=210,000 \mathrm{MPa}$. Different Poisson's ratios $v$ are to be set in different simulations.

\section{Numerical model results vs. analytical model}

1) Unidimensional horizontal tension test $\sigma_{x 0}=20 \mathrm{Mpa}$;

The strain values in three radial directions have been investigated in the experimental hole drilling method. As the displacement field determines the strain and stress field, only the displacement field is necessary to be validated. For the circular structure, as the $\theta=\pi / 2$ is a special case of $\theta=0$, only two directions are necessary to be taken for the validation as shown in Figure 3 and Figure 4.

The cylindrical displacements $u_{r}, u_{\theta}$ for different value of Poisson's ratio $v$ in $\theta=0$ direction have been shown in Figure 3. $u_{r}$ obtained by analytical and FE method are almost the same. $u_{\theta}$ are zero for both analytical and FE method.

The cylindrical displacements $u_{r}, u_{\theta}$ for different value of Poisson's ratio $v$ in $\theta=\pi / 4$ direction have been shown in Figure 4. Very close results obtained by analytical and FE method have been found for both $u_{r}$ and $u_{\theta}$.

All the non-zero displacements in Figure 3 and in Figure $\mathbf{4}$ have the maximum value at the drilled surface and converge to zero at the outer bound.

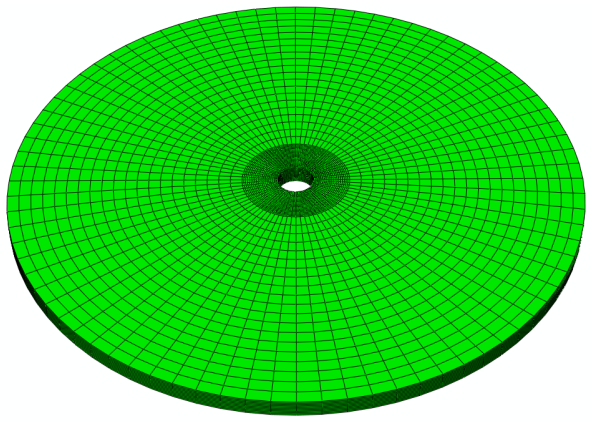

Figure 2. Round plate and hole used in hole-drilling method.
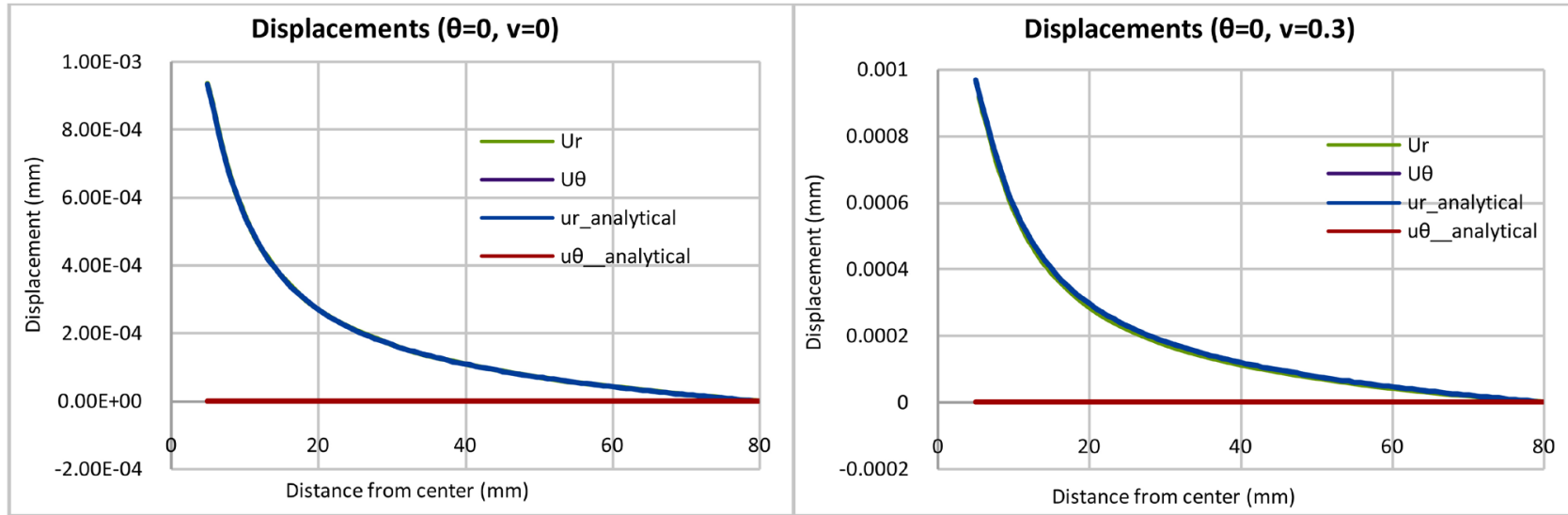

Figure 3. Displacements for different $v$ in $\theta=0$ direction. 
2) Shearing test $\tau_{x y 0}=20 \mathrm{Mpa}$

The displacement distributions in two directions are shown in Figure $\mathbf{5}$ and Figure 6. Very close results have been found by the analytical and FE method for the shearing test.

Based the above observation, the best place to put the strain gages are near the hole.
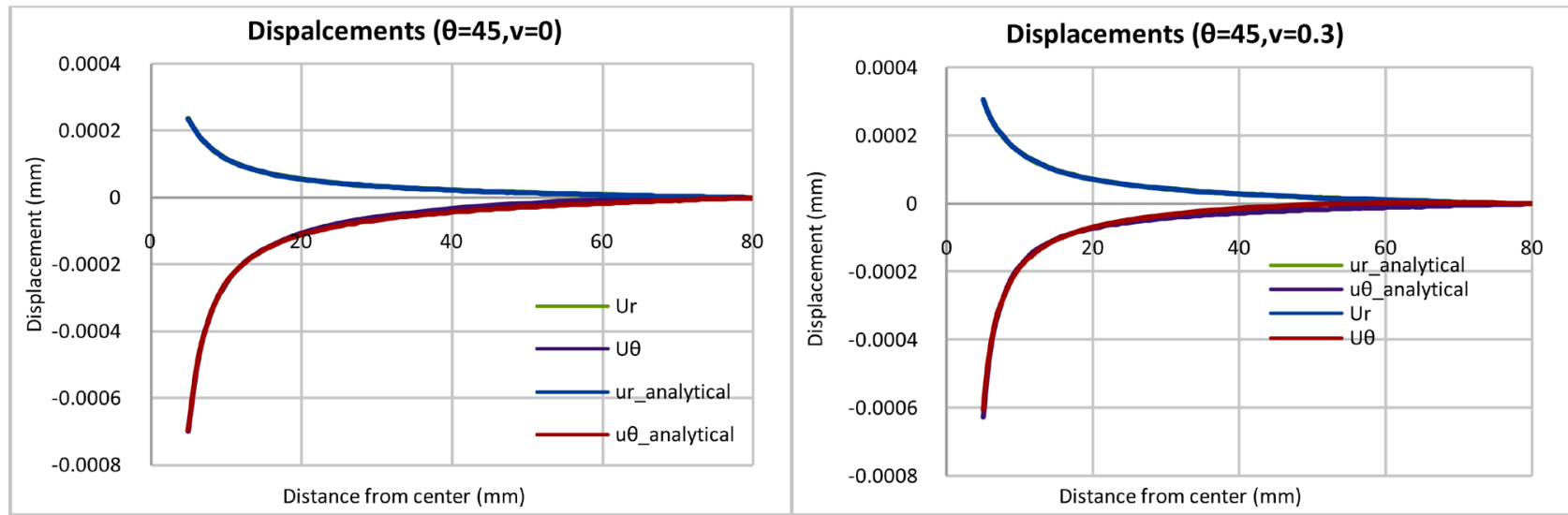

Figure 4. Displacements for different $v$ in $\theta=\pi / 4$ direction.
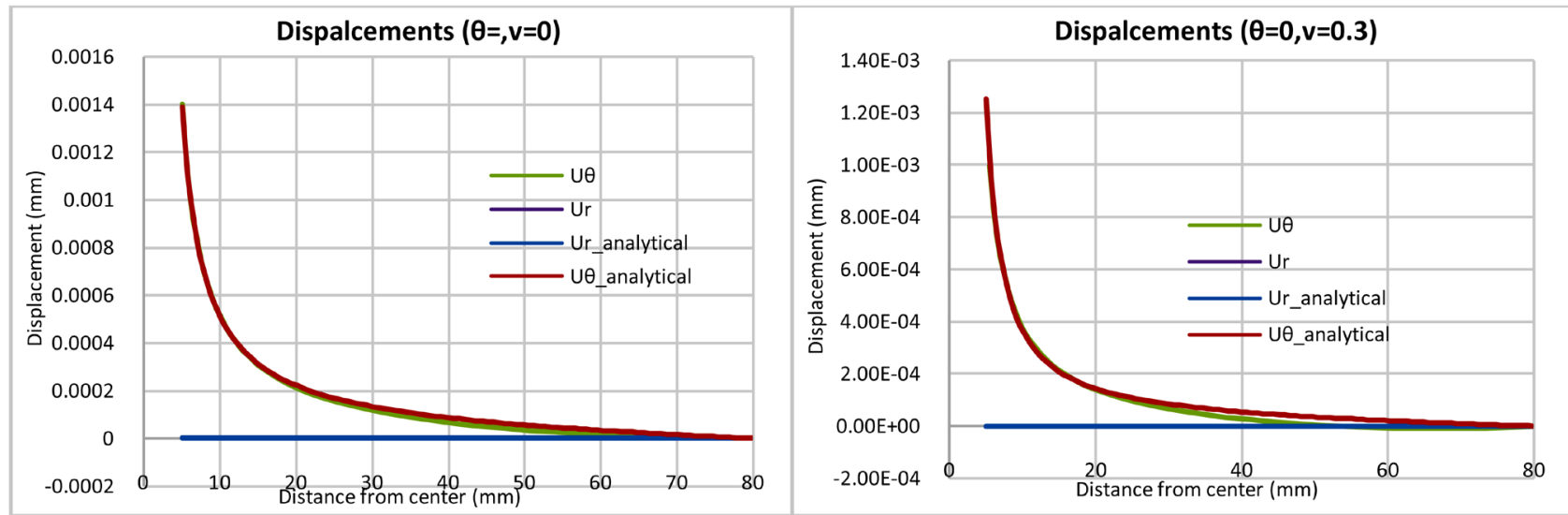

Figure 5. Displacements for different $v$ in $\theta=0$ direction.
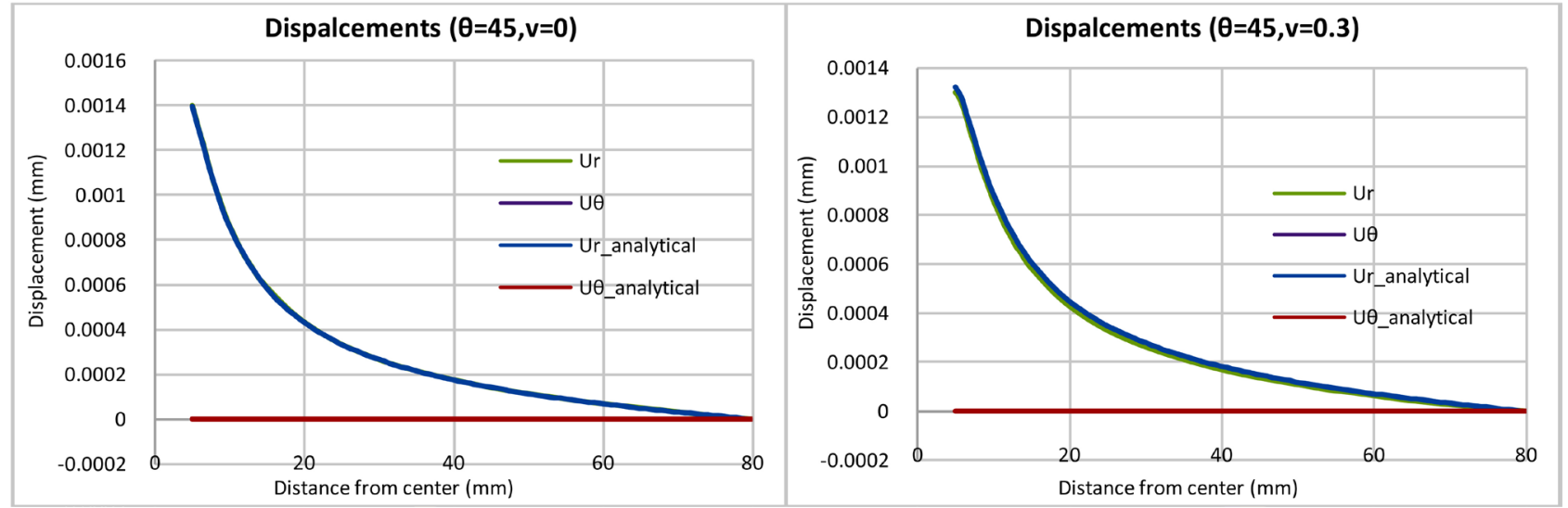

Figure 6. Displacements for different $v$ in $\theta=\pi / 4$ direction. 


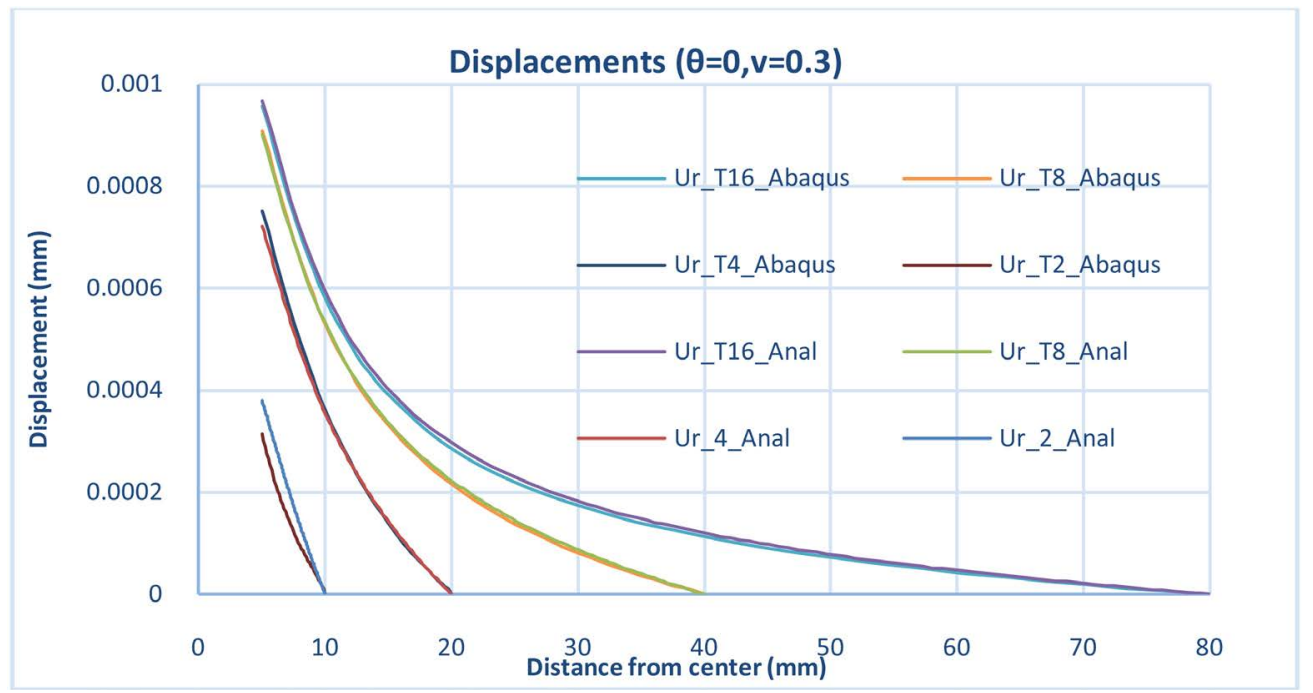

Figure 7. Displacements for $v=0.3$ in $\theta=0$ direction

3) Unidimensional horizontal tension test $\sigma_{x 0}=20 \mathrm{Mpa}$ with different diameter

In order to show the present analytical solution is suitable for thin plate to satisfy the plane stress hypothesis, different diameters of the plate are studied. The unidimensional tension test $\sigma_{x 0}=20 \mathrm{Mpa}$ is used.

Figure 7 shows that when the ratio between the plate's diameter and the drilling hole diameter $\lambda(T)=8$ is good enough for the analytical model.

\section{Conclusions}

A displacement field based analytical model to determine the residual stress components in a finite elastic thin plate with hole-drilling method has been developed.

The proposed analytical model uses the finite boundary conditions that are realist and it has been validated by FE simulations. The obtained displacement fields in different directions for pure tensile and pure shearing are in very good agreement with those obtained by shell FE simulations (Abaqus). Theoretically this model keeps the capacity to calculate the residual stress components for an infinite plate.

This proposed model enables the determination of residual stress components in a thin finite elastic plate to be fulfilled with an efficient analytical solution without using FE simulations that are time-consuming.

The future work will be carried out by using incremental hole-drilling method in a composite plate including different displacement field in $\mathrm{z}$ direction where the stress based model cannot be used.

\section{Conflicts of Interest}

The authors declare no conflicts of interest regarding the publication of this paper. 


\section{References}

[1] Rendler, N.J. and Vigness, I. (1966) Hole-Drilling Strain-Gage Method of Measuring Residual Stresses. Experimental Mechanics, 6, 577-586.

[2] Kabiri (1986) Toward More Accurate Residual-Stress Measurement by the Hole-Drilling Method: Analysis of Relieved-Strain Coefficients. Experimental Mechanics, 26, 14-21.

[3] Beghini, M. and Bertini, L. (1998) Recent Advances in the Hole Drilling Method for Residual Stress Measurement. Journal of Materials Engineering and Performance, 7, 163-172.

[4] Youngdahl, C.K. and Sternberg, E. (1966) Three-Dimensional Stress Concentration around a Cylindrical Hole in a Semi-Infinite Elastic Body. Journal of Applied Mechanics, December, 855-865.

[5] Grinchenko, V.G. and Ulitko, A.F. (1970) An Exact Solution of the Kirsch Problem. Soviet Applied Mechanics, 6, 455-461.

[6] David Roylance (2001) Closed-Form Solutions.

https://ocw.mit.edu/courses/materials-science-and-engineering/3-11-mechanics-ofmaterials-fall-1999/modules/MIT3_11F99_airy.pdf 